\title{
Infection after Constrained Condylar Knee Arthroplasty: Incidence and microbiological findings in 100 consecutive complex primary and revision total knee arthroplasties
}

\author{
Marianne Westberg ${ }^{\bowtie}$, Bjarne Grøgaard, Finnur Snorrason \\ Division of Orthopaedic Surgery, Oslo University Hospital, Oslo, Norway \\ $\bowtie$ Corresponding author: Marianne Westberg, M.D. PhD, Telephone: +47 22118080; E-mail address: marianne.westberg@oslo-universitetssykehus.no \\ (c) Ivyspring International Publisher. This is an open access article distributed under the terms of the Creative Commons Attribution (CC BY-NC) license \\ (https://creativecommons.org/licenses/by-nc/4.0/). See http://ivyspring.com/terms for full terms and conditions.
}

Received: 2018.07.31; Accepted: 2018.10.31; Published: 2018.12.08

\begin{abstract}
Background: To report incidence, microbiological findings, and treatment outcome of prosthetic joint infection (PJI) after constrained condylar knee arthroplasty.

Methods: Retrospective study of 100 consecutive complex primary and revision total knee arthroplasties operated with constrained condylar knee arthroplasties between February 2006 and October 2015 at a single institution. Demographic and surgical data were registered, as well as data regarding infection, microbiology and treatment. Follow-up median 43 months; SD 32.

Results: The overall incidence of acute postoperative PJI was low (3/100). 1/33 (3\%), 1/ 45 (2\%), and $1 / 22$ (5\%), following primary complex TKA, aseptic revisions, and septic revisions, respectively. The incidence of late acute hematogenous PJI was 5/100. 1/33 (3\%), 1/45 (2\%), and 3/22 (14\%) following primary complex TKA, aseptic revisions, and septic revisions, respectively. Late acute hematogenous infections were associated with a lower success rate of treatment with debridement and implant retention compared with acute postoperative PJls, $1 / 5$ versus 2/3.

Conclusion: The risk of late acute hematogenous infections seems increased. Multiple previous surgery, poor soft tissue, and large metal implants may contribute to an increased lifelong susceptibility for bacteremias to cause PJIs.
\end{abstract}

Key words: Total knee arthroplasty, condylar constrained, revision surgery, complex primary, prosthetic joint infection, microbiology

\section{Introduction}

Prosthetic joint infection (PJI) is one of the most feared and challenging complications after total knee arthroplasty (TKA), leading to reoperations, longer hospitalizations, and increased morbidity, mortality and health care costs. The incidence of PJI after primary TKA is approximately $1-2 \%[1,2]$. Infection after revision TKAs occurs more frequently, reported up to $7-9 \%[3,4,5]$. In recent years, PJI is reported by several to be the leading cause of re-revision after revision TKA $[6,7,8,9]$.
Traditionally, constrained and hinged TKAs have been reserved for revision settings with excessive bone loss and instability [10]. However, constrained implants are currently more commonly used in all types of revision TKAs, and also in primary TKAs with ligamentous deficiency or severe deformities $[10,11,12,13]$. The number of primary and revision TKAs has increased substantially over the past decades, and the volume, including revisions, is expected to continue to rise, especially in young 
patients $[14,15]$.

Risk factors for PJI following primary TKAs are identified and reported by several. They include male gender, fracture sequelae, impaired wound healing, and the use of constrained implants $[1,16,17]$. The literature on revision TKA is more sparse, and it is difficult to interpret due to small and heterogenous patient materials. Many of the patient series reported are on aseptic revisions only, and very few on complex primary constrained TKAs. Consequently, little is therefore known on risk factors for infection after constrained and hinged TKAs.

This study was performed to determine the incidence, the microorganism causing PJIs, and outcome of infection in patients operated with constrained and hinged TKAs as a primary or revision procedure.

\section{Materials and Methods}

We retrospectively studied 100 (92 patients) Rotating Hinge Knee prosthesis (RHK) and Constrained Condylar Knee prosthesis (LCCK) (NexGen; ZimmerBiomet Inc., Warsaw, IN, USA) that were implanted in a consecutive series of revision and complex primary TKAs at a single tertiary referral center between February 2006 and October 2015.

Factors known as potential predictors of infection were recorded, both patient-related and surgery-related factors: age, gender, American Society of Anesthesiology (ASA) score, Body Mass Index (BMI), smoking, diabetes, immuno-suppressive therapy, diagnosis at the time of index TKA, length of time between primary and revision TKA, previous surgical procedures, previous infections and indication for revisions. Surgical factors were type of implant, use of pneumatic tourniquet, operation time, surgical drains, the use of gentamicin-containing fleeces and how the wound was closed. The main baseline patient characteristics are reported in Table 1 and data on surgical methods are shown in Table 2. An overview of the indications for the revision TKAs is presented in Table 3.

The surgery was performed using standard technique with a midline incision in primary cases and in the previous scar in revisions. In case of multiple scars, the most lateral was used in order to decrease the risk of skin necrosis. Parapatellar arthrotomy was used in the majority of cases, but a tibial tubercle osteotomy was used in cases of difficult exposure (Table 2). The indication for a hinged prosthesis was severe instability with absence or disruption of ligaments. The semi constrained LCCK was used in cases with intermediate insufficiency of the ligaments and/or moderate bone loss. There were some changes in the surgical procedures over the years. During most of the study period, it was standard procedure to use a pneumatic tourniquet, either during the whole operation or under the prosthesis fixation. This procedure was abandoned in 2013. Likewise, surgical drainage for 24 hours and admission of gentamicin collagen fleeces were frequently used initially, but only occasionally the last three years of the study period. The wound was closed with staples in the initial 4 years of the period, then the routine was changed to sutures. All patients received prophylactic antibiotics; intravenous cephalothin 2 grams times 4 . In the revisions, and in primary cases where there had been previous surgical procedures or infections, the prophylaxis was withheld until microbiological biopsies were obtained. Cement fixation with gentamicin-containing cement was utilized for the tibial and femoral components. In case of patella resurfacing, a cemented all-poly button was used.

In the 22 patients undergoing septic revision, a two-stage procedure was performed in all cases. A gentamicin containing cement spacer was placed in the period between removal of the prosthesis and reimplantation.

Table 1. Preoperative patient characteristics in 100 constrained and hinged condylar TKAs

\begin{tabular}{lll}
\hline Characteristics & Primary TKA (n=33) & Revision TKA (n=67) \\
\hline Age, median years (SD) & $64(15)$ & $64(9,9)$ \\
Sex, female n (\%) & $18(54)$ & $31(46)$ \\
Comorbidities, n (\%) & & \\
ASA-score 1-2 & $22(67)$ & $48(72)$ \\
ASA-score 3 & $11(33)$ & $19(28)$ \\
BMI, median (SD) & $26(3,7)$ & $29(5,9)$ \\
Diabetes & $1(3)$ & $9(13)$ \\
Smoking & $5(15)$ & $9(13)$ \\
Immuno supressed & $3(9)$ & $4(6)$ \\
Diagnosis index TKA, n (\%) & & \\
Idiopathic osteoarthritis & $4(12)$ & $49(73)$ \\
Post-traumatic osteoarthritis & $22(67)$ & $16(24)$ \\
Neuromuscular instability & $3(9)$ & \\
Congential malalignment & $3(9)$ & \\
Septic arthritis & $1(3)$ & $1(1,5)$ \\
Inflammatory arthritis & & $1(1,5)$ \\
Seq. patellectomy & & \\
\hline ASA = American Society of Anesthesiology, BMI = Body Mass Index $\left(\mathrm{kg} / \mathrm{m}^{2}\right)$
\end{tabular}

Table 2. Surgical data

\begin{tabular}{lll}
\hline & Primary TKA (n=33) & Revision TKA (n=67) \\
\hline $\begin{array}{l}\text { Surgery time, median min. (SD) } \\
\text { Type of implant, } \mathbf{n}(\%)\end{array}$ & $211(63.9)$ \\
LCCK & $11(33)$ & $47(70)$ \\
RHK & $22(67)$ & $20(30)$ \\
Surgical details, $\mathbf{n}(\%)$ & & \\
Patella component & $7(21)$ & $35(52)$ \\
Tourniquet & $9(27)$ & $6(9)$ \\
Tibial tubercle osteotomy & $1(3)$ & $19(28)$ \\
Surgical drain & $12(36)$ & $20(30)$ \\
Sutures vs staples & $27(82)$ vs $6(18)$ & $59(88)$ vs $8(12)$ \\
Intraarticular gentamicin mats & $8(24)$ & $20(30)$
\end{tabular}

LCCK = Legacy constrained condylar knee, RHK = rotating hinge knee 
Table 3. Reasons for revision in 67 revision TKAs

\begin{tabular}{ll}
\hline Reason & Number of revisions, $\mathbf{n}(\mathbf{\%})$ \\
\hline Infection & $22(33)$ \\
Loosening & $18(27)$ \\
Instability & $14(21)$ \\
Malrotation & $3(4.5)$ \\
Patella problems & $1(1.5)$ \\
Stiffness & $1(1.5)$ \\
Other & $8(11)$ \\
\hline
\end{tabular}

Infection was diagnosed clinically based on the CDC definition of deep surgical site infection [18]. PJI was classified according to Segawa; (1) Positive intraoperative culture; (2) early postoperative infection (< 4 weeks postoperatively); (3) late chronic infection; (4) acute hematogenous infection [19].

The treatment strategy for PJI was soft tissue debridement with retention of the implant and changing of the polyethylene liner in patients with early PJI or suspected acute hematogenous PJI (DAIR procedure: debridement, antibiotics, irrigation, and retention). The DAIR procedure was followed by two weeks of intravenous antimicrobial therapy, then four to six weeks of treatment per mouth. An empirical intravenous antimicrobial regimen consisting of vancomycin in combination with a $\beta$-lactam, was started perioperatively and maintained until definitive microbiological results were known. Organismspecific antibiotics were then given. Further suppressive therapy was not given after the treatment period of 6-8 weeks. If this treatment failed, or in late infections, a two stage procedure was performed. This procedure included removal of the prosthesis and cement, and a thorough soft tissue debridement. Antibiotic treatment was administered intravenously for two weeks, followed by typically six to eight weeks of treatment per mouth. The patients underwent a minimum of 14 days off antibiotics before reimplantation.

\section{Statistics}

Descriptive statistics were computed with SPSS for Windows version 23.0 (SPSS Inc, Chicago, IL, USA). The chi-squared test and Fischer's Exact test were used to compare categorical variables, while t-tests were used for continuous variables. Due to the low number of infections, we were not able to conduct statistical analyses on risk factors for infection or treatment failure. A p-value of $<0.05$ was considered significant.

\section{Results}

Thirty-three implants were used as a primary TKA whereas 67 were total revisions. Of the revisions, 45 were for aseptic failure and 22 revisions were reimplantations following a two-stage procedure for
PJI. The patients were scheduled for an active follow-up at 3 months, one year and then according to the surgeons preference. The minimum follow-up was one year (median 43 months; SD 32). All patients were seen at one year with the exception of one patient who died in-hospital 11 months postoperative of other causes with a well-functioning TKA. In case of complications with the TKA, the patients were routinely readmitted. With the exception of 8 procedures, all operations were performed by the same four experienced knee surgeons. The hospital's Data Protection Official for Research approved the study.

Overall, PJI developed in 9 knees (7 patients) (Table 4). Three of these infections were early infections, five were regarded as acute hematogenous infections occuring after median 1129 days (range; 546-1579), and one infection was a chronic infection following reimplantation.

Table 4. Overview of infections

\begin{tabular}{|c|c|c|c|}
\hline & Early acute PJI & $\begin{array}{l}\text { Acute hematogenous } \\
\text { PJI }\end{array}$ & $\begin{array}{l}\text { Late chronic } \\
\text { PJI }\end{array}$ \\
\hline $\begin{array}{l}\text { Primary knees } \\
n=33\end{array}$ & $1 / 33(3 \%)$ & $1 / 33(3 \%)$ & - \\
\hline $\begin{array}{l}\text { Aseptic revision } \\
\mathrm{n}=45\end{array}$ & $1 / 45(2 \%)$ & $1 / 45(2 \%)$ & - \\
\hline $\begin{array}{l}\text { Septic revision } \\
\mathrm{n}=22\end{array}$ & $1 / 22(5 \%)$ & $3 / 22(14 \%)$ & $1 / 22(5 \%)$ \\
\hline Microbial agent & $\begin{array}{l}\text { Staph aur; } n=2 \\
\text { Staph epi; } n=1\end{array}$ & $\begin{array}{l}\text { Streptococci; } n=3 \\
\text { Staph aur; } n=2\end{array}$ & Staph epi; $\mathrm{n}=1$ \\
\hline Treatment & DAIR; $n=3$ & DAIR; $n=5$ & $\begin{array}{l}\text { Suppressive } \\
\text { therapy }\end{array}$ \\
\hline Outcome & $\begin{array}{l}\text { Success; } n=2 \\
\text { Failure; } n=1\end{array}$ & $\begin{array}{l}\text { Success; } n=1 \\
\text { Failure; } n=4\end{array}$ & $\begin{array}{l}\text { Chronic } \\
\text { infection }\end{array}$ \\
\hline
\end{tabular}

\section{Complex primary TKAs}

Thirty-two patients (33 knees) were operated with a primary LCCK $(n=11)$ or RHK $(n=22)$. Eighteen of the patients (56\%) were female, and the majority of procedures were due to trauma $(22 / 33 ; 67 \%)$. Only four of these patients had primary osteoarthritis as cause of TKA. 21/33 (64\%) had had median 1 (0-13; SD 2,9) pre-arthroplasty surgical procedure(s). These procedures included ligament reconstruction, fracture surgery, reconstruction of vascular injury, fasciotomy, reconstruction of soft tissue injury, arthroscopic debridement, high tibial osteotomy, fixation hardware removal, and meniscectomy. Three of the patients had a history of previous infection in the knee, all caused by Staphylococcus aureus. None of the patients had growth from perioperatively obtained biopsies.

Deep infection developed in two knees in the same patient. The right knee was the first to be operated and initially without problems. Then the left knee was operated a few months later. An early PJI occurred due to Staphylococcus aureus and group G 
streptococci. A soft tissue debridement was performed after 42 days, and there were no signs of infection after 5 years follow-up. In the first knee operated, an acute hematogenous infection occurred after 1200 days due to an acute hematogenous infection caused by Staphylococcus aureus. A DAIR procedure was performed, but without success. The prosthesis later had to be removed in a two-stage procedure. This patient had an immunodeficiency disorder, and had infections following several other surgical procedures.

\section{Revision TKAs}

In the 67 revisions, 36 (54\%) TKAs were in male patients. Primary osteoarthitis was the diagnosis for the index TKA in the majority of patients (73\%). 39 $(58 \%)$ of the patients had had a median of $1(0-8$; SD $1,5)$ surgical procedure(s) before the index arthroplasty: fracture surgery, meniscectomy, high tibial osteotomy, ligament reconstruction, patellectomy, soft tissue revisions, hardware removal, and minor arthroscopic procedures. A median of 2 (1-7; SD 1,5) previous arthroplasty-procedures were performed, including removal of the prosthesis.

Fourty-five $(67 \%)$ of the revisions were aseptic revisions, and loosening and instability were the main causes for revision (Table 3). Four of the patients undergoing aseptic revision had a history of infection in an earlier knee procedure, caused by streptococci $(n=1)$ and Staphylococcus aureus $(n=3)$, but none of these developed PJI after the revision TKA. None of the 45 patients grew bacteria from the perioperative biopsies. PJI after aseptic revision TKA developed in 2 of $45(4 \%)$ patients. One patient developed an early PJI due to Staphylococcus aureus, and a soft tissue debridement was performed after 39 days. This patient died within a year of other causes, but with no signs of infection. One patient developed an acute hematogenous infection with group $G$ streptococci, and underwent a soft tissue debridement after 546 days. The patient then had to go through heart surgery, which also got infected, but by another microbe. He eventually had to remove the knee prosthesis due to treatment failure in a two-stage procedure.

Twenty-two (33\%) of the revision procedures (21 patients) were reimplantations after previous PJI treated by a two-stage procedure. Sixteen $(76 \%)$ of the patients were male. The initial PJI was mainly caused by Coagulase-negative staphylococci (CoNS) (57\%) and Staphylococcus aureus (9\%). At the most recent follow-up, five knees were reinfected, of which two reinfections were in the same patient and in the same knee. With the exception of one patient who developed a chronic infection with Staphylococcus epidermidis and then treated with suppressive antimicrobial treatment, the infections were reoperated with a soft tissue revision after median 693 (range; 22-1129) days. One patient had an early postoperative infection with Staphylococcus epidermidis, and three had an acute haematogenous infection caused by Group $\mathrm{G}$ streptococci $(\mathrm{n}=2)$, and Staphylococcus aureus $(\mathrm{n}=1)$. None of the reinfections were caused by the same microorganism that was identified in the initial septic revision. One hematogenous infection with Group G streptococci was successfully treated with DAIR, but 3 of 4 reinfections had to be further revised in a two-stage procedure.

\section{Discussion}

The early postoperative infection rate in this patient material with 100 LCCKs and RHKs was promising, with $1 / 33$ infections in complex primary TKAs, and $1 / 45$ in aseptic revisions. The use of constrained and hinged prosthesis has been identified as a predictor of infection $[17,20]$. The patients where RHK and LCCK were used as a primary TKA, were often operated because of posttraumatic arthrosis and many of the patients had gone through several previous surgical procedures. These are also factors known to increase the risk of PJI $[4,17,21]$.

The infection rate after septic revision is over fivefold higher than the infection rate following aseptic revisions. Perioperative biopsies were obtained during all aseptic revisions, of which none were positive. This strengthens the diagnosis of asepsis, and the infection rate following aseptic revisions in this study $(2 / 45,4 \%)$ was satisfactory compared to previous studies reporting 9-10\% [4]. This may be due to a thorough preoperative work up ensuring that a low grade chronic infection not was mistaken for aseptic loosening.

A two-stage procedure has traditionally been regarded as the preferred treatment in chronic PJI following knee arthroplasty, but good infections control has in more recent literature also been reported following a one-stage procedure [22,23]. The two-stage procedure is still often referred to as the gold standard. The reinfection rate is however still high, reported in previous studies to be $16-28 \%$ $[24,25]$. Our data $(5 / 22,23 \%)$ is in accordance with this, but the failure rate seems to be in the upper range. The results could be explained by the type of infections. Our center is a tertiary referral center, and difficult infections are frequently referred from other institutions. This could also be due to definition of failure. In our series, none of the patients with success were treated with long term suppressive antibiotics. Interestingly, none of the reinfections were caused by 
the initial infecting organism. This is also reported by others, and Zmistowski et al. have suggested that in many cases, recurrent PJI is in fact a new infection, and that host risk factors are more important than difficulties in eradicating the infection [26]. Further, previous infection is by several been identified as a risk factor for PJI $[4,17,27]$. This could be explained by repeated surgery and compromised soft tissues, by overlooked chronic infections, or maybe by genetic or other host factors still unknown.

In the literature, acute hematogenous PJIs are considered to be quite rare, compared to the acute postoperative and chronic infections, but the incidence appears to be increasing and is also reported higher for knees than for hips [28]. In this material, the numbers of acute hematogenous infections were surprisingly high (5/9) at the latest follow-up. In comparison, Cholewinski et al. reported a high infection rate $(9,3 \%)$ in a cohort of 43 primary LCCKs, of which $3 / 4$ were delayed infections [29]. It is challenging to be sure whether an acute hematogenous infection is in fact an acute infection or just an exacerbation of a chronic infection. Chronic infections may also debut suddenly without weeks or months with symptoms prior to clinical manifestation. The knees in this material were all clinically uneventful until an acute inset of infection after median 3 years postoperative, and we therefor find the diagnosis most likely to be acute hematogenous infections. Likewise, we think that the high number of streptococcal infections also strengthens the diagnosis of acute hematogenous infections. Predisposing risk factors like multiple previous surgery compromizing the soft tissue around the knee joint, and large metal implants may contribute to an increased susceptibility for acute hematogenous PJIs.

The failure rate of surgical treatment was higher among the acute hematogenous infections than the acute postoperative infections. The results of soft tissue debridement in acute hematogenous infections are also previous reported to be poorer than in early post operative infections [30]. Streptococci have historically been regarded as an easy-to-treat microbe in PJIs [31,32]. This was not the case in our series. Worse prognosis in streptococcal infections has recently also been shown by others, and the ideal treatment for streptococcal PJIs may yet to be established [33,34].

In conclusion, the early postoperative infection rate in this material of 100 constrained TKAs was promising, but it appears to be a high risk of acute hematogenous PJIs associated with unfavourable results after DAIR. Compromized soft tissues combined with large metal implants may contribue to an increased lifelong susceptibility for bacteremias to cause PJIs.

\section{Author contribution}

M Westberg: Initiated the study, collected data, performed analysis and wrote the manuscript. B Grøgaard: Contributed to the study design, drafting and reviewing the manuscript. F Snorrason: Contributed to the study design, drafting and reviewing the manuscript.

\section{Competing Interests}

The authors have declared that no competing interest exists.

\section{References}

[1] Pulido L, Ghanem E, Joshi A, Purtill J J, Parvizi J. Periprosthetic joint infection: the incidence, timing, and predisposing factors. Clin Orthop Relat Res 2008; 466(7): 1710-1715.

[2] Kurtz SM, Ong KL, Lau E, Bozic KJ, Berry DJ. Prosthetic Joint Infection Risk after TKA in the Medicare Population. Clin Orthop Relat Res 2010; 468(1): 52-56.

[3] Sheng P Y, Jamsen E, Letho M, Pajamaki J, Halonen P, Konttinen Y T. Revision total knee arthroplasty with the total condylar III system: a comparative analysis of 71 consecutive cases of osteoarthritis or inflammatory arthritis. Acta Orthop 2006; 77(3): 512-518.

[4] Mortazavi S M, Schwartzenberger J, Austin M S, Purtill J J, Parvizi J. Revision total knee arthroplasty infection:Incidence and predictors. Clin Orthop Relat Res 2010; 468(8): 2052-2059.

[5] Luque R, Rizo B, Urda A, Garcia-Crespo R, Moro E, Marco F, Lopez-Duran L. Predective factors for failure after total knee replacement revision. Int Orthop 2014; 38(2): 429-435.

[6] Suarez J, Griffin W, Springer B, Fehring T, Mason J B, Odum S. Why do revision knee arthroplasties fail? J Arthroplasty 2008; 23(6 Suppl 1): 99-103.

[7] Hossain F, Patel S, Haddad F S. Midterm assessment of causes and results of revision total knee arthroplasty. Clin Orthop Relat Res 2010; 468: 1221-1228.

[8] Bozic K J, Kurtz S M, Lau E, Ong K, Chiu V, Vail T P, Rubash H E, Berry D J. The epidemiology of revision total knee arthroplasty in the United States. Clin Orthop Relat Res 2010; 468(1): 45-51.

[9] Leta T H, Lygre S H L, Skredderstuen A, Hallan G, Furnes O. Failure of aseptic revision total knee arthroplasties. 145 revision failures from the Norwegian Arthroplasty Register, 1994-2011. Acta Orthopaedica 2015; 86(1): 48-57.

[10] Barrack R L. Rise of the rotating hinge in revision total knee arthroplasty. Orthopedics 2002; 25(10): 1020-1058.

[11] Gudnason A, Milbrink J, Hailer N P. Implant survival and outcome after rotating-hinge total knee revision arthroplasty: a minimum 6-year follow up. Arch Orthop Trauma Surg 2011; 131: 1601-1607.

[12] Maynard L M, Sauber T J, Kostopoulos V K, Lavigne G S, Sewecke J J, Sotereanos N G. Survival of primary condylar-constrained total knee arthroplasty at a minimum of 7 years. J Arthroplasty 2014; 29: 1197-1201.

[13] Baker P, Critchley R, Gray A, Jameson S, Gregg P, Port A, Deehan D. Mid-term survival following primary hinged total knee replacement is good irrespective of the indication for surgery. Knee Surg Sports Traumatol Arthrosc 2014; 22: 599-608.

[14] Kurtz S M, Ong K, Lau E, Mowat F, Halpern M. Projections of primary and revision hip and knee arthroplasty in the United States from 2005 to 2030. J Bone Joint Surg Am 2007; 89(4): 780-785.

[15] Kurtz S M, Lau E, Ong A, Zhao K, Kelly M, Bozic K J. Future young patient demand for primary and revision joint replacement: national projections from 2010 to 2030. Clin Orthop Relat Res 2009; 467: 2606-2612.

[16] Saleh K, Olson M, Resig S, Bershadsky B, Kuskowski M, Gioe T, Robinson H, Schmidt R, McElfresh E. Predictors of wound infection in hip and knee joint replacement: results from a 20 year surveillance program. J Orthop Res 2002; 20: 506-515.

[17] Jamsen E, Huhtala H, Puolakka T, Moilanen T. Risk factors for infection after knee arthroplasty. A register-based analysis of 43,149 cases. J Bone Joint Surg Am 2009; 91(1): 38-47.

[18] Mangram A J, Horan T C, Pearson M L, Silver L C, Jarvis W R. Guideline for Prevention of Surgical Site Infection, 1999. Centers for Disease Control and Prevention (CDC) Hospital babkinInfection Control Practices Advisory Committee. Am J Infect Control 1999; 27(2): 97-132.

[19] Segawa H, Tsukayama D T, Kyle R F, Becker D A, Gustilo R B. Infection after total knee arthroplasty. A retrospective study of the treatment of eighty-one infections. J Bone Joint Surg Am 1999; 81(10): 1434-1445. 
[20] Babkin $Y$, Raveh D, Lifschitz M, Itzchaki M, Wiener-Well Y, Kopuit P, Jerassy $\mathrm{Z}$, Yinnon AM. Incidence and risk factors for surgical infection after total knee replacement. Scand J Infect Dis 2007; 39(10): 890-895.

[21] Peersman G, Laskin R, Davis J, Peterson M. Infection in total knee replacement: a retrospective review of 6489 total knee replacements. Clin Orthop Relat Res 2001;392: 15-23.

[22] Zahar A, Kondoff DO, Klatte TO, Gehrke TA. Can good infection control be obtained in one-stage exchange of the infected TKA to a rotating hinge design? 10 years results. Clin Orthop Relat Res 2016; 474(1): 81-87.

[23] Haddad FS, Sukeik M, Alazzawi S. Is single-stage revision according to a strict protocol effective in treatment of chronic knee arthroplasty infections? Clin Orthop Realt Res 2015; 473(1): 8-14.

[24] Mortazavi S M, Vegari D, Ho A, Zmistowski B, Parvizi J. Two-stage exchange arthroplasty for infected total knee arthroplasty: predictors of failure. Clin Orthop Relat Res 2011; 469(11): 3049-3054.

[25] Kubista B, Hartzler R U, Wood C M, Osmon D R, Hanssen A D, Lewallen D G. Reinfection after two-stage revision for periprosthetic infection of total knee arthroplasty. Int Orthop 2012; 36(1): 65-71.

[26] Zmistowski B, Tetreault MW, Alijanipour P, Chen AF, Della Valle CJ, Parvizi J. Recurrent periprosthetic joint infection. Persistent or new infection? J Arthroplasty 2013; 28: 1486-1489.

[27] Bongartz T, Halligan CS, Osmon DR, Reinalda MS, Bamlet WR, Crowson CS, Hanssen AD, Matteson EL. Incidence and risk factors of prosthetic joint infection after total hip or knee replacement in patients with rheumatoid arthritis. Arthritis Rheum 2008; 59(12): 1713-1720.

[28] Huotari K, Peltola M, Jamsen E. The incidence of late prosthetic joint infections. A registry-based study of 112,708 primary hip and knee replacements. Acta Orthopaedica 2015; 86(3): 321-325.

[29] Cholewinski P, Putman S, Vasseur L, Migaud H, Duhamel A, Behal H, Pasquier G. Long-term outcomes of primary constrained condylar knee arthroplasty. Orthop Traumatol Surg Res 2015; 101: 449-454.

[30] Vilchez F, Martinez-Pastor JC, Garcia-Ramiro S, Bori G, Tornero E, Garcia E, Mensa J, Soriano A. Efficacy of debridement in hematogenous and early post-surgical prosthetic joint infections. Int J Artif Organs 2011;34(9): 863-869.

[31] Odum SM, Fehring TK, Lombardi AV, Zmitowski BM, Brown NM, Luna ST, Fehring KA, Hansen EN. Irrigation and debridement for periprosthetic infections: does the organism matter? J Arthroplasty 2011; 26(6 Suppl): 114-118.

[32] Betz M, Abrassart S, Vaudaux P, Gjika E, Schindler M, Billieres J, Zenelaj B, Suvà D, Peter R, Uckay I. Increased risk of joint failure in hip prosthesis infected with Staphylococcus aureus treated with debridement, antibiotics and implant retention compared to Streptococcus. Int. Orthop 2015;39(3):397-401.

[33] Lora-Tamayo J, Senneville E, Ribera A, Bernard L, Dupon M, Zeller V, et al. The not-so-good prognosis of Streptococcal periprosthetic joint infection managed by implant retention: The results of a large multicenter study. Clin Infect Dis 2017;64(12):1742-1752.

[34] Akgün D, Trampuz A, Perka C, Renz N. High failure rates in treatment of Streptococcal periprosthetic joint infection: results from a seven-year retrospective cohort study. Bone Joint J 2017; 99B(5):653-659. 